\title{
Consumption of human rabies immunoglobulin (HRIG) in government hospitals of Sri Lanka.
}

\author{
WDNL Amarasinghe ${ }^{1}$, O Wimalaratne ${ }^{1}$, S Nanayakkara ${ }^{1}$, TKGS Sumathipala ${ }^{1}$, \\ MGK de Costa ${ }^{2}$
}

Sri Lankan Journal of Infectious Diseases 2016 Vol.6 (2):114-118

DOI: http://dx.doi.org/10.4038/sljid.v6i2.8114

\begin{abstract}
Introduction

Rabies still remains as a significant public health problem in Sri Lanka. Published data related to the usage of human rabies immunoglobulins (HRIG) which is an expensive biological used for post exposure prophylaxis and the types of animal bites are limited. This study was designed to analyze the consumption of HRIG for major animal bites in government hospitals of Sri Lanka.
\end{abstract}

\section{Methods}

An audit was carried out at the Rabies Unit in the Medical Research Institute (MRI) on the use of HRIG from 1st of January 2010 to 31st of December 2012. Data was extracted from scrutinizing the forms sent to the Rabies Unit following the administration of HRIG by the government hospitals which administer HRIG.

\section{Results}

1896, 2160, 2186 patients received HRIG during 2010, 2011 and 2012 respectively. The number of patients treated without adhering to the rabies treatment protocol issued by the ministry of health also increased over the same period of time. Each year, around $60 \%$ of total HRIG has been used for stray dog and cat bites, $24 \%$ for domestic animal bites and $17 \%$ for wild animal bites.

\section{Conclusions and recommendations}

Annual consumption as well as the misuse of HRIG has increased from 2010 to 2012. Effective methods to control stray dogs and cats and the concept of responsible pet ownership should be encouraged. Medical officers in rabies treatment units should be trained on adherence to government protocol for anti- rabies post exposure therapy.

Keywords : rabies, immunoglobulin, HRIG, animal bites

\footnotetext{
${ }^{1}$ Medical Research Institute, Colombo 8, Sri Lanka

${ }^{2}$ Military Hospital, , Horton Pl, Colombo 7, Sri Lanka

Address for correspondence: Dr. K Nanayakkara, Consultant Virologist/Vaccinologist, Medical Research Institute, Colombo 8, Sri Lanka Email: ; kanthinana@yahoo.com Phone: + 94777715839
}

Receivede 27 May 2016 and revised version accepted 17 October 2016 


\section{Introduction}

Rabies remains a major public health problem in Sri Lanka. In order to protect people with major exposures ${ }^{1}$ from developing rabies, those considered to be at risk are treated immediately with anti-rabies immunoglobulins (Human / Equine), followed by a course of anti-rabies vaccine as per WHO recommendation. ${ }^{1}$

Human rabies immunoglobulin (HRIG) is a biological in limited supply, and is considerably more expensive than equine rabies immunoglobulin. The Government of Sri Lanka spends over 50 million US\$ for the entire rabies control programme annually. ${ }^{2}$ HRIG is administered according to body weight and the cost is approximately 200 US $\$$ for an average $60 \mathrm{~kg}$ adult. The Ministry of Health has issued strict guidelines and implemented a protocol on the use of HRIG for anti-rabies post exposure therapy when treating major exposures to ensure appropriate use and prevent misuse. ${ }^{3}$

HRIG is currently available in government hospitals, but limited to Teaching Hospitals and a few General and Base hospitals to minimize irrational usage. It is important to audit the usage of HRIG and compliance to the protocol with the aim of taking appropriate action if necessary to minimize incorrect usage in the future.

In 2007, a decision was made by the government against elimination of stray dogs. It is therefore important to know the fraction of HRIG used for major exposures following stray dog bites. ${ }^{3}$

There are no published Sri Lankan data on major exposures according to the type of biting animal including wild animal bites. This retrospective analysis will also show the types of wild animal bites for which HRIG has been used in the 3 years of the study.

The main objective of the study was to analyze the consumption of human rabies immunoglobulin in government hospitals of Sri Lanka according to the number of patients treated and to observe the pattern of usage during the period 2010-2012. The study would also determine the percentage of human rabies immunoglobulin used inappropriately island wide and in each hospital during the study period. In addition, the usage of HRIG according to the habitat and type of the biting animal would also be analyzed. .

\section{Methods}

The audit was conducted at the Department of Rabies \& Vaccine Quality Control, Medical Research Institute (MRI). The MRI receives all patient information forms for mandatory surveillance following the administration of HRIG in government hospitals. Details of the exposure, type and status of the biting animal and details of HRIG used are entered in these forms by the administering hospital staff. A total of 6242 forms received from $1^{\text {st }}$ January 2010 to $31^{\text {st }}$ December 2012 were analyzed in MS-Excel according to the objectives of this study.

Scientific and ethical approval to conduct the research was obtained from the Research and Ethics Committee of the MRI. 


\section{Results}

A total of 6242 patients received HRIG during this three year period with 1896, 2160 and 2186 patients receiving HRIG during 2010, 2011and 2012 respectively. HRIG was administered without proper adherence to the government protocol during each year in 60 $(3 \%), 103(5 \%)$ and $170(8 \%)$ patients respectively (Figure 1).

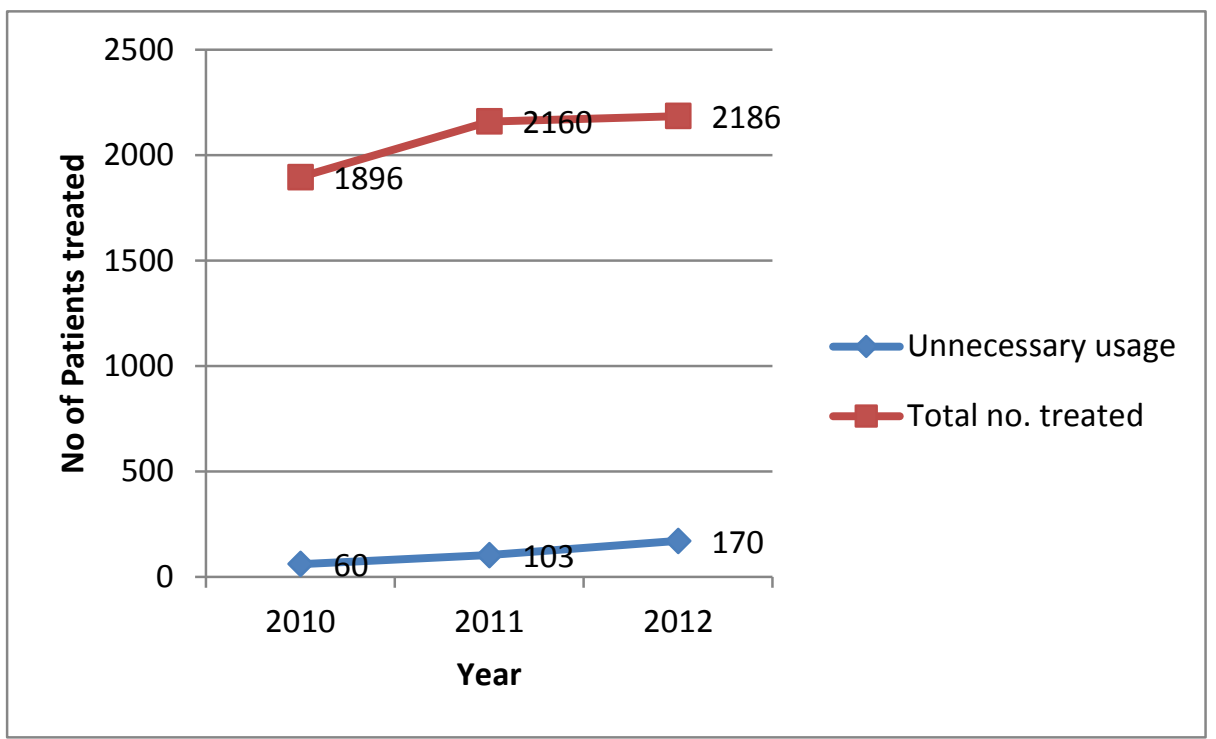

Figure 1 : Total annual consumption and total annual unnecessary usage of HRIG $(2010-2012)$

A total of 13 government hospitals, including all Teaching Hospitals and a few General and Base hospitals were administering HRIG by 2012. The percentage of HRIG used unnecessarily in each hospital was separately analyzed. Of 4 hospitals to which HRIG was recently introduced (during 2011 and 2012), 3 showed >50\%, unnecessary usage while the other

Table 1: Categorization of major bites for which HRIG hospital showed $<30 \%$ given according to the habitat of the animal.

\begin{tabular}{cccccccc}
\hline Year & \multicolumn{2}{c}{ Stray } & \multicolumn{2}{c}{ Domestic } & \multicolumn{2}{c}{ Wild } & Total \\
\hline $\mathbf{2 0 1 0}$ & 1095 & $58 \%$ & 484 & $26 \%$ & 315 & $16 \%$ & 1894 \\
$\mathbf{2 0 1 1}$ & 1312 & $61 \%$ & 488 & $23 \%$ & 356 & $16 \%$ & 2156 \\
$\mathbf{2 0 1 2}$ & 1257 & $58 \%$ & 500 & $23 \%$ & 425 & $19 \%$ & 2182 \\
\hline
\end{tabular}

During this period, total HRIG of $58-61 \%$ has been used for stray dog and cat bites, $23-26 \%$ for domestic dog and cat bites and $16-19 \%$ for wild animal bites (Table 1). Annually, around $68 \%$ of HRIG has been used on dog bites. Patients with bandicoot, monkey, mongoose and squirrel bites were the most common wild animal bites presenting during this period (Table 2). 
Table 2: Type of animal bite which required HRIG during 2010-2012.

\begin{tabular}{|c|c|c|c|c|c|c|c|c|c|c|c|c|}
\hline $\begin{array}{l}\bar{\Xi} \\
\stackrel{\Xi}{\Xi}\end{array}$ & $\begin{array}{l}0 \\
60 \\
0^{\circ}\end{array}$ & $\stackrel{\tilde{E}}{\tilde{E}}$ & $\begin{array}{l}\overrightarrow{8} \\
: \overrightarrow{0} \\
\tilde{\Xi} \\
\oplus\end{array}$ & 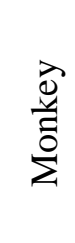 & $\begin{array}{l}0 \\
8 \\
8 \\
80 \\
\tilde{0} \\
\tilde{\Sigma}\end{array}$ & 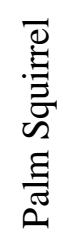 & 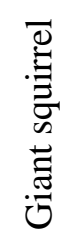 & 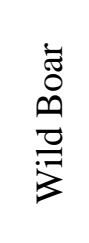 & 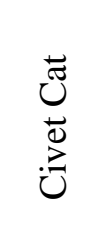 & $\begin{array}{l}\tilde{J} \\
0 \\
0 \\
0\end{array}$ & 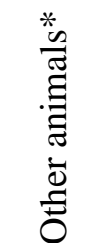 & 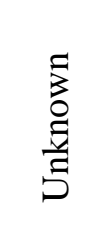 \\
\hline \multirow[t]{2}{*}{2010} & 1320 & 258 & 69 & 54 & 56 & 42 & 35 & 6 & 9 & 17 & 12 & 18 \\
\hline & $70 \%$ & $14 \%$ & $4 \%$ & $3 \%$ & $3 \%$ & $2 \%$ & $2 \%$ & $0.3 \%$ & $0.4 \%$ & $0.8 \%$ & $0.6 \%$ & $0.9 \%$ \\
\hline \multirow[t]{2}{*}{2011} & 1496 & 337 & 57 & 67 & 40 & 47 & 61 & 8 & 3 & 17 & 10 & 17 \\
\hline & $69 \%$ & $16 \%$ & $3 \%$ & $3 \%$ & $2 \%$ & $2 \%$ & $3 \%$ & $0.4 \%$ & $0.1 \%$ & $0.8 \%$ & $0.5 \%$ & $0.8 \%$ \\
\hline \multirow[t]{2}{*}{2012} & 1498 & 322 & 57 & 61 & 32 & 80 & 60 & 18 & 8 & 25 & 12 & 13 \\
\hline & $87 \%$ & $15 \%$ & $3 \%$ & $3 \%$ & $1 \%$ & $4 \%$ & $3 \%$ & $1 \%$ & $0.4 \%$ & $1 \%$ & $0.5 \%$ & $0.6 \%$ \\
\hline
\end{tabular}

*Other animals included cattle, bat, pig, raccoon, rat, fox, hamster, goat, bear, fishing Cat, rabbit, horse, deer, donkey, otter and porcupine

\section{Discussion}

An increase in the total HRIG consumption in government hospitals of Sri Lanka from 2010 to 2012 was observed in this study, with an increasing trend of administration of HRIG without following the guidelines in the protocol. However, island wide correct usage of HRIG each year still remains $>90 \%$ and contributes to the success of the anti-rabies programme. A high percentage of unnecessary HRIG use was observed in centres with recent introduction of HRIG administration. The cause of this requires further investigation. However, it is possible that excess use reflects inaccurate decisions made by the inexperienced healthcare staff

The majority of HRIG administration was used for stray animal bites (58-61\%) throughout all three years, reflecting the inadequacy of existing stray animal control programmes in Sri Lanka. In Sri Lanka as well as in the world ${ }^{4}$, the main reservoir of rabies is considered as dogs and more attention should be paid specially in the control of the stray dog population. Usage of HRIG for domestic animals (23-26\%) can be minimized by proper adherence to strategies of responsible pet ownership. The presentation following wild animal bites in Sri Lanka differ from some other countries where raccoon, skunk, bat and fox bites are common. ${ }^{5}$

\section{Recommendations and conclusion}

Compliance to the post-exposure protocol issued by the ministry of health is high at $90 \%$. However, existing noncompliance could be reduced by ensuring strict compliance to the current protocol in the administration of HRIG before a decision is made on rabies postexposure prophylaxis. It is important to provide regular continuous medical educational (CME) programmes to medical staff managing patients who seek rabies post exposure therapy from government hospitals in order to maximize compliance and minimize wastage of HRIG.

The finding that approximately $60 \%$ of animal bites requiring HRIG are from stray animals, requires revisiting the current stray animal control programme so that appropriate feasible 
alternatives be explored. The concept of responsible pet ownership among the Sri Lankan public should also be promoted, which would result in a reduction of persons who truly require rabies post-exposure therapy.

Repeat audits should be done periodically to reassure the success and cost effectiveness of the rabies control programme.

\section{References}

1 WHO Expert consultation on Rabies. $2^{\text {nd }}$ report. Geneva, WHO. 2013.

2 Director General of Health Services. Circular 2016-127 Revised protocol for antirabies postexposure therapy; 2016 accessed at http://www.mri.gov.lk/assets/Rabies/Revised-Protocool-16.03.2016.pdf

2 Rabies in Asia Foundation. $3^{\text {rd }}$ Rabies in Asia Conference: RIACON. 2011.

3 Balasubramaniam R, Kasturiratne A, Wimalaratne O, Wickremasinghe A.R, Comparison of number of dog rabies diagnosed at the Medical Research Institute before and after stray dog elimination cessation policy. The Journal of the College of Community Physicians.2013; Supplement1:35 accessed at http://hdl.handle.net/123456789/11415

4 World Organization of Animal Health, World Health Organization, Food and Agriculture Organization of the United Nations. Rationale for investing in the global elimination of dog-mediated human rabies. 2015; 37.

5 CDC-Rabies,(Cited 2016 May 23); accessed at http://www.cdc.gov/rabies/ 